\title{
Dermatofibrosarcoma Protuberans of the Neck: A Case Report
}

\author{
Tzu-I Wu1, Hsing-Mei Wu',2, Yih-Jeng Tsai1,2, Tzu Hsuan Luo', Chia-Jung Lee1,2 \\ ${ }^{1}$ Department of Otolaryngology Head and Neck Surgery, Shin Kong Wu-Ho-Su Memorial Hospital, Taiwan \\ ${ }^{2}$ School of Medicine, Fu-Jen Catholic University, Taiwan \\ Email: M013973@ms.skh.org.tw
}

How to cite this paper: $\mathrm{Wu}, \mathrm{T} .-\mathrm{I}, \mathrm{Wu}$, H.-M., Tsai, Y.-J., Luo, T.H. and Lee, C.-J. (2021) Dermatofibrosarcoma Protuberans of the Neck: A Case Report. International Journal of Otolaryngology and Head \& Neck Surgery, 10, 85-91.

https://doi.org/10.4236/ijohns.2021.102009

Received: January 12, 2021

Accepted: March 1, 2021

Published: March 4, 2021

Copyright $\odot 2021$ by author(s) and Scientific Research Publishing Inc. This work is licensed under the Creative Commons Attribution International License (CC BY 4.0).

http://creativecommons.org/licenses/by/4.0/

\begin{abstract}
Background: Dermatofibrosarcoma protuberans (DFSP) is rare soft tissue sarcoma but has a locally aggressive nature. Although most cases are of low grade and have a rare metastasis rate, all DFSP variants have a tendency to show local recurrence. Wide excision with negative margins is the treatment of choice. Aim: To report a case of DFSP who presented with an asymptomatic slow growing tumor similar to etiologies such as hypertrophic scars or other benign soft tissue tumors. Case Presentation: A 68-year-old male presented with a large soft tumor located at the left posterior neck. Local excision was done under the preoperative impression of a benign tumor such as lipoma or sebaceous cyst. However the diagnosis of DFSP was made upon histological examination and the patient underwent another surgery to achieve free resection margins under general anesthesia as well as adjuvant radiotherapy. Conclusion: DFSP is a malignant tumor that is diagnosed histopathologically. Due to the low incidence rate, slow-growing nature, and non-alarming initial presentation features, diagnostic delay or even misdiagnosis is not uncommon.
\end{abstract}

\section{Keywords}

Dermatofibrosarcoma Protuberans, Soft Tissue Sarcoma, Surgery

\section{Introduction}

Dermatofibrosarcoma protuberans (DFSP) is an uncommon locally aggressive cutaneous soft tissue sarcoma of the dermis layer of the skin [1] with an incidence of 0.8 - 4.5 cases per million per year [2] [3]. Approximately 85 to 90 percent of DFSPs are low grade. Despite its rare metastasis rate $(<5 \%)$, all DFSP variants have a tendency to show local recurrence [4] [5]. Most commonly occurring in adult patients in their thirties, DFSP accounts for approximately $1 \%-6 \%$ 
of soft tissue sarcoma cancers [5] [6] [7]. More than 90\% of DFSP tumors have rearrangement of chromosome 17 and 22 that fuses collagen gene COL1A1 with platelet-derived growth factor (PDGF), leading to transcriptional up-regulation of PDGF and thus established a self-stimulatory growth signal [7]. Immunoreactivity for CD34 in DFSP was described in 1993 and remains to be the main immunohistochemical marker for diagnosing DFSP [7]. Due to its slow growing nature similar to nodules that appear as hypertrophic scars or benign soft tissue tumors without any definite symptoms, it is difficult to diagnose pre-operatively. The preferred initial treatment for a localized dermatofibrosarcoma protuberans (DFSP) is resection with pathologically negative margins. However, extensive excision may not be feasible due to either anatomic limitation or cosmetic concerns, especially if found in the head and neck region. A case requiring an additional surgery and adjuvant radiotherapy, due to difficulty of an accurate preoperative diagnosis and tumor location, is reported here.

\section{Case Report}

A 68-year-old Taiwanese man visited our clinic due to noticing a left posterior neck mass for 6 months. The patient reported progressive growth of the tumor in size during the preceding six months. The patient denied any recent weight loss, fever, night sweats or chills. On physical examination, the mass measured about $2.0 \times 2.0 \mathrm{~cm}$ over the patient's left posterior neck, and it was firm, painless, with no sign of localized heat or erythematous change, and relatively movable. The patient's neck was supple, with no palpable cervical lymphadenopathies. The patient denied past history of malignancy. A neck sonography exam was performed, and a well-defined, heterogeneous subcutaneous tumor was noted, measuring $1.58 \times 0.92 \mathrm{~cm}$, with posterior enhancement (Figure 1). Under the impression of a posterior neck lipoma or sebaceous cyst, the patient underwent excisional biopsy under local anesthesia on an outpatient basis. Gross tumor was completely removed, but an infiltrative nature was noted around the border without a well-defined margin. Upon histological examination, infiltrating and hypercellular tumor composed of interlacing fascicles of hyperchromatic atypical spindle cells arranged in vague storiform fashion was described (Figure 2), as well as abnormal mitotic figures average 4 per 10 HPFs noted. Special stain showed tumor cells are immunoreactive against antibody to CD34 (Figure 3). Based on the histological and immunohistochemical findings, the diagnosis of dermatofibrosarcoma was made. The patient underwent an additional wide excision under general anesthesia. Due to lack of gross tumor, the wide excision was focused to encompass the whole previous surgical site with resection margins checked intra-operatively via frozen section pathologic review. Although the histopathology report revealed free margins and only few atypical spindle cells present in the specimen, the surgeon felt that resection of an adequate margin could not be achieved, especially the deep margin, due to the location of the tumor being at the neck. The patient underwent adjuvant local radiotherapy treatment with a total radiation dose of $66 \mathrm{~Gy}$ in 33 fractions. The patient cur- 
rently has no signs of local recurrence 12 months after surgery, and is under regular follow-up with sonography exam (Figure 4).

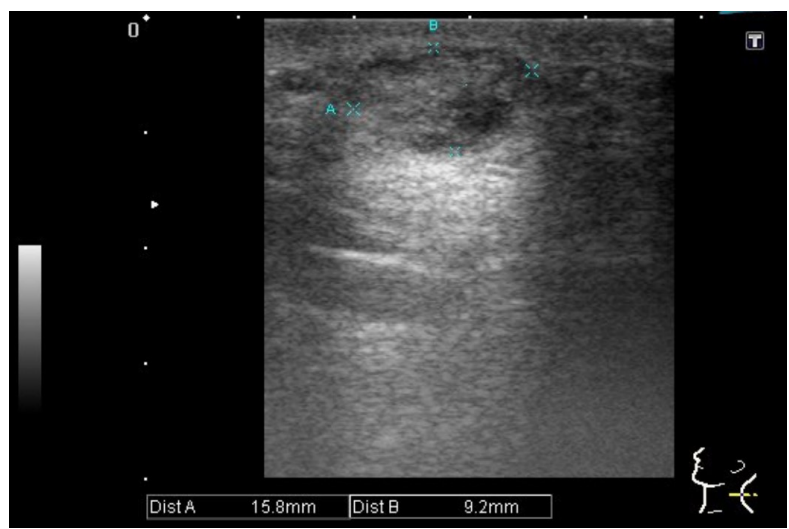

Figure 1. Pre-operative sonography of the posterior neck lesion showing a relatively well-defined heterogeneous nodular lesion with acoustic shadow.

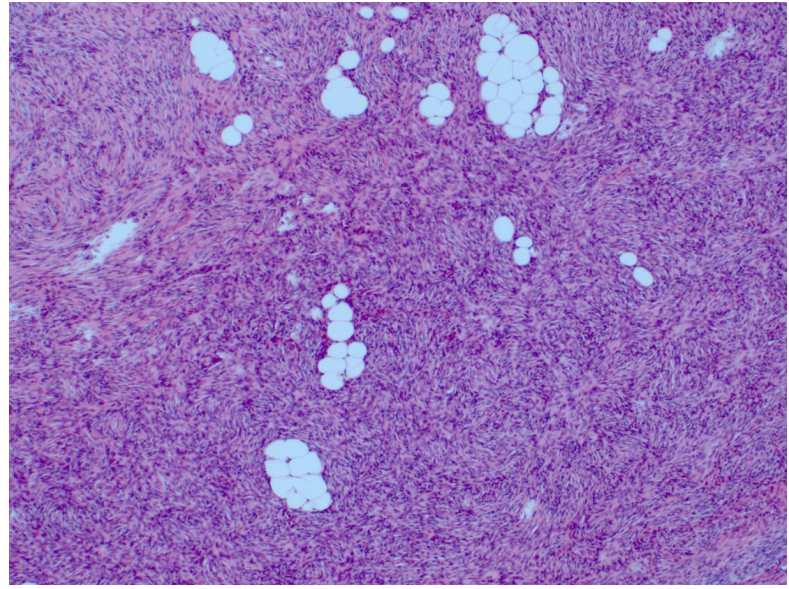

Figure 2. Histological examination: infiltrating and hypercellular tumor composed of interlacing fascicles of hyperchromatic atypical spindle cells arranged in vague storiform fashion.

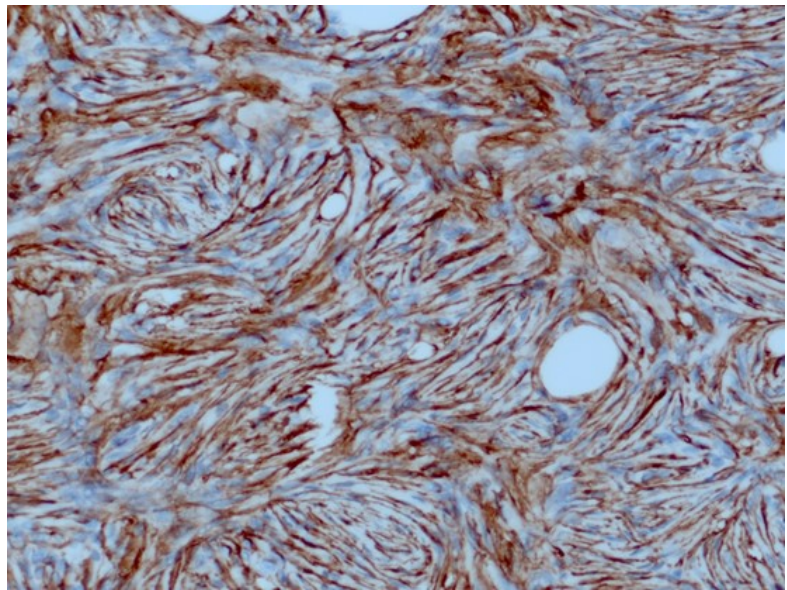

Figure 3. Special staining showed the tumor is immunoreactive against antibody to $\mathrm{CD} 34$. 


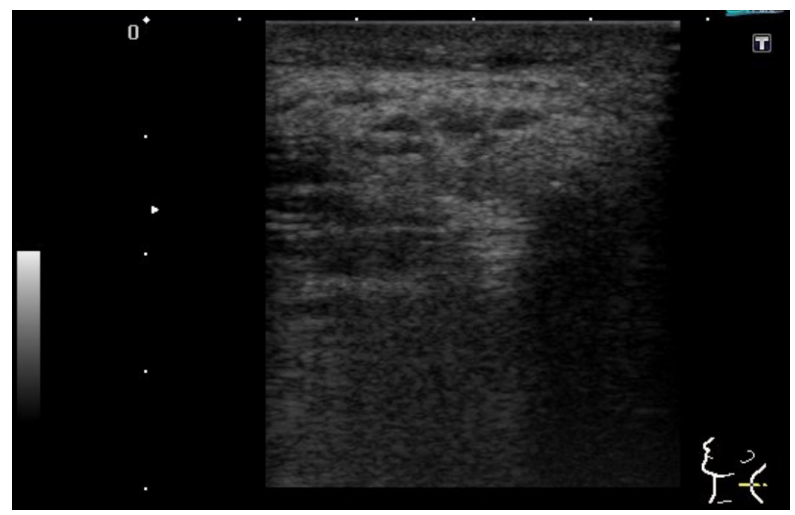

Figure 4. Post-operative sonography examination done 12 months after surgery showing no tumor recurrence.

\section{Discussion}

In the early stages, it is difficult to differentiate DFSP from other benign subcutaneous lesions such as lipomas, epidermal cysts, keloids, or dermatofibroma without undergoing surgical excision and histology examination. DFSP often presents as a minor skin firm area. It commonly resembles a bruise or pimple, and is a slow growing tumor. The most common location of DFSP is the trunk $(42 \%-72 \%)$ followed by proximal extremities $(20 \%-30 \%)$, with head and neck region being the least $(10 \%-16 \%)$ [8].

With sonography, DFSPs have been found to be around (67\%) or ovoid (33\%) subcutaneous lesion that are mostly well defined (89\%) [9]. Appearance of DFSP usually involves a mildly lobulated border and a heterogeneously hypoechoic matrix. Posterior enhancement can also usually be noted [9]. Vascularity of DFSP, which is a marker of malignancy, varies as well. Since lipomas may also present with similar features, a distinction is not always possible. Hence, an initial preoperative impression of a benign lesion such as sebaceous cyst or lipoma was made for our case, which led to additional surgery and adjuvant treatment. Several authors have reported benefits of utilizing magnetic resonance imaging (MRI) preoperatively to better evaluate tumor extent [10]. In cases of large tumors ( $>4 \mathrm{~cm}$ in diameter), MRI is able to aid clinicians in distinguishing the relationship of the tumor with surrounding structures [11].

Definite diagnosis of DFSP requires histopathological examination. In order to ensure adequate tissue for examination, biopsy should be performed with a core-needle or surgical incision [12]. Microscopically, DFSP infiltrates the dermis diffusely, while often sparing the epidermis and skin. Kim et al. described seven histological DFSP subtypes, with "classic" DFSP comprising about 90\% 13 . The "classic" subtype of DFSP has histological characteristics of monomorphous fusiform cells (spindle cells) and a large elongated nucleus and a low mitotic index. These spindle cells are irregularly organized with a storiform arrangement [13]. Due to its tendency to invade surrounding tissue from a central lesion, DFSP has projections that show a honeycomb (30\%) or multilayered $(70 \%)$ subcutaneous pattern [14] [15]. DFSP typically shows positive staining for vi- 
mentin, CD34, apolipoprotein D, and nestin on immunohistochemistry [16].

DFSP is a low-grade malignancy, but has been reported a local recurrence rate up to $60 \%$ that may be attributed to incomplete excision due to poor circumscription and irregular boundaries [17]. Wide excision with $2-4 \mathrm{~cm}$ safe margin remains the cornerstone of the treatment of DFSP [12] [18] [19]. Although a sufficient excision margin is the key to reduce the local recurrence rate, it is often limited by the anatomical site, and the lesion typically infiltrates well beyond its grossly visible margin into the surrounding tissue. The use of Mohs micrographic surgery with incremental excision until normal tissue is obtained, as documented by repeat frozen sections, has been favored as the treatment of choice by some authors [20] [21].

Given that metastases to lymph nodes are extremely rare, there is no role for prophylactic regional node dissection. Radiotherapy (RT) is a noninvasive adjuvant treatment that can improve local control in patients, especially those having close or positive margins and re-excision not feasible. Several studies have suggested post-operative adjuvant RT reduced recurrence rate. A retrospective study of 184 patients reported a significant 5-year disease-free survival improvement in patients receiving adjuvant RT compared with patients that did not $(88.1 \%$ vs $56.2 \%, P=0.044)$ [22]. In our case, re-excision surgery was done after the diagnosis was made in an effort to achieve free and adequate resection margins. Although free surgical margin was reported, a wide safe margin was not feasible due to the location of the tumor, and adjuvant RT was utilized to improve local control. For DFSP patients with inoperable or metastatic diseases, Imatinib therapy is currently the standard of care. This targeted therapy may also potentially facilitate resection or decrease possible disfigurement when used in a neoadjuvant fashion [23].

\section{Conclusion}

DFSP is a rare locally aggressive malignant soft tissue sarcoma that originates from the dermis layer of the skin. Diagnostic delay or even misdiagnosis is not uncommon due to its indolent nature. DFSP is diagnosed by histopathology examination; therefore a biopsy or at least a core-needle biopsy is required in order to ensure adequate tissue specimen is obtained. Wide excision with negative margins is the treatment of choice. For patients with close or positive surgical margins, RT is a noninvasive adjuvant treatment that improves local control.

\section{Acknowledgements}

This research received no specific grant from any funding agency, commercial or not-for-profit sectors.

\section{Conflicts of Interest}

The authors declare no conflicts of interest regarding the publication of this paper. 


\section{References}

[1] Eguzo, K., Camazine, B. and Milner, D. (2014) Giant Dermatofibrosarcoma Protuberans of the Face and Scalp: A Case Report. International Journal of Dermatology, 53, 767-772. https://doi.org/10.1111/j.1365-4632.2012.05639.x

[2] Bhambri, S., Desai, A., Del Rosso, J.Q. and Mobini, N. (2008) Dermatofibrosarcoma Protuberans: A Case Report and Review of the Literature. Journal of Clinical and Aesthetic Dermatology, 1, 34-36.

[3] Criscione, V.D. and Weinstock, M.A. (2007) Descriptive Epidemiology of dermatofibrosarcoma Protuberans in the United States, 1973 to 2002. Journal of the American Academy of Dermatology, 56, 968-973. https://doi.org/10.1016/j.jaad.2006.09.006

[4] McArthur, G. (2007) Dermatofibrosarcoma Protuberans: Recent Clinical Progress. Annals of Surgical Oncology, 14, 2876-2886. https://doi.org/10.1245/s10434-007-9480-y

[5] Sanmartín, O., Llombart, B., López-Guerrero, J.A., Serra, C., Requena, C. and Guillén, C. (2007) Dermatofibrosarcoma Protuberans [Dermatofibrosarcoma Protuberans]. Actas Dermo-Sifiliográficas, 98, 77-87. https://doi.org/10.1016/S0001-7310(07)70019-4

[6] Kransdorf, M.J. (1995) Malignant Soft-Tissue Tumors in a Large Referral Population: Distribution of Diagnoses by Age, Sex, and Location. American Journal of Roentgenology, 164, 129-134. https://doi.org/10.2214/ajr.164.1.7998525

[7] Llombart, B., Serra-Guillén, C., Monteagudo, C., López Guerrero, J.A. and Sanmartín, O. (2013) Dermatofibrosarcoma Protuberans: A Comprehensive Review and Update on Diagnosis and Management. Seminars in Diagnostic Pathology, 30, 13-28. https://doi.org/10.1053/j.semdp.2012.01.002

[8] Stivala, A., Lombardo, G.A., Pompili, G., Tarico, M.S., Fraggetta, F. and Perrotta, R.E. (2012) Dermatofibrosarcoma Protuberans: Our Experience of 59 Cases. Oncology Letters, 4, 1047-1055. https://doi.org/10.3892/ol.2012.887

[9] Shin, Y.R., Kim, J.Y., Sung, M.S. and Jung, J.H. (2008) Sonographic Findings of Dermatofibrosarcoma Protuberans with Pathologic Correlation. Journal of Ultrasound in Medicine, 27, 269-274. https://doi.org/10.7863/jum.2008.27.2.269

[10] Torregiani, W.C., Al-Ismail, K., Munk, P., Nicolaou, S., O’Connell, J.X. and Knowling, M.A. (2002) Dermatofibrosarcoma Protuberans: MR Imaging Features. American Journal of Roentgenology, 178, 989-993. https://doi.org/10.2214/ajr.178.4.1780989

[11] Riggs, K., McGuigan, K.L., Morrison, W.B., Samie, F.H. and Humphreys, T. (2009) Role of Magnetic Resonance Imaging in Perioperative Assessment of Dermatofibrosarcoma Protuberans. Dermatologic Surgery, 35, 2036-2041.

https://doi.org/10.1111/j.1524-4725.2009.01330.x

[12] National Comprehensive Cancer Network (2019) NCCN Clinical Practice Guidelines in Oncology (NCCN Guidelines ${ }^{\mathrm{TM}}$ ) Dermatofibrosarcoma Protuberans Version 1. https://www.nccn.org/professionals/physician gls/PDF/dfsp.pdf

[13] Kim, G.K. (2011) Status Report on the Management of Dermatofibrosarcoma Protuberans: Is There a Viable Role for the Use of Imatinib Mesylate? In Which Cases May It Be Therapeutically Helpful and in Which Cases Not? Journal of Clinical and Aesthetic Dermatology, 4, 17-26.

[14] Kamino, H. and Jacobson, M. (1990) Dermatofibrome Extending into the Subcutaneous Tissu: Differential Diagnosis from Dermatofibrosarcoma Protuberans. The American Journal of Surgical Pathology, 14, 1156-1164. 
[15] Zelger, B.W., Ofner, D. and Zelger, B.G. (1995) Atrophic Variants of Dermatofibroma and Dermatofibrosarcoma Protuberans. Histopathology, 26, 519-527. https://doi.org/10.1111/j.1365-2559.1995.tb00270.x

[16] Paramythiotis, D., Stavrou, G., Panagiotou, D., Petrakis, G. and Michalopoulos, A (2016) Dermatofibrosarcoma Protuberans: A Case Report and Review of the Literature. Hippokratia, 20, 80-83.

[17] Chang, C.K., Jacobs, I.A. and Salti, G.I. (2004) Outcomes of Surgery for Dermatofibrosarcoma Protuberans. European Journal of Surgical Oncology, 30, 341-345. https://doi.org/10.1016/j.ejso.2003.12.005

[18] Dubay, D., Cimmino, V., Lowe, L., Johnson, T.M. and Sondak, V.K. (2010) Low Recurrence Rate after Surgery for Dermatofibrosarcoma Protuberans: A Multidisciplinary Approach from a Single Institution. Cancer, 100, 1008-1016. https://doi.org/10.1002/cncr.20051

[19] Stojadinovic, A., Karpoff, H.M., Antonescu, C.R., Shah, J.P., Singh, B., Spiro, R.H., et al. (2000) Dermatofibrosarcoma Protuberans of the Head and Neck. Annals of Surgical Oncology, 7, 696-704. https://doi.org/10.1007/s10434-000-0696-3

[20] Dim-Jamora, K.C. and Perone, J.B. (2008) Management of Cutaneous Tumors with Mohs Micrographic Surgery. Seminars in Plastic Surgery, 22, 247-256. https://doi.org/10.1055/s-0028-1095884

[21] Ratner, D., Thomas, C.O., Johnson, T.M., Sondak, V.K., Hamilton, T.A., Nelson, B.R., et al. (1997) Mohs Micrographic Surgery for the Treatment of Dermatofibrosarcoma Protuberans. Results of a Multiinstitutional Series with an Analysis of the Extent of Microscopic Spread. Journal of the American Academy of Dermatology, 37, 600-613. https://doi.org/10.1016/S0190-9622(97)70179-8

[22] Du, K., Li, J., Tang, L., Lin, X., Kong, X., Liao, X., Peng, Q., Dong, Y., He, J., Huang, Y., Zhang, X., Lin, F., Zhuang, Q. and Wu, J. (2019) Role of Postoperative Radiotherapy in Dermatofibrosarcoma Protuberans: A Propensity Score-Matched Analysis. Radiation Oncology, 14, Article No. 20. https://doi.org/10.1186/s13014-019-1226-Z

[23] Lemm, D., Mügge, L.O., Mentzel, T. and Höffken, K. (2009) Current Treatment Options in Dermatofibrosarcoma Protuberans. Journal of Cancer Research and Clinical Oncology, 135, 653-665. https://doi.org/10.1007/s00432-009-0550-3 